\title{
Errata - É importante saber onde posicionar o marcador esférico para o planejamento digital de artroplastia de quadril?
}

Giancarlo Cavalli Polesello ${ }^{1}$ Thiago Tronco Salerno ${ }^{1}$ João Hélio Zucarelli Rezende ${ }^{1}$ Marcelo Cavalheiro de Queiroz ${ }^{1}$ Nayra Deise Rabelo ${ }^{1,2}$ Walter Ricioli Junior ${ }^{1}$

\footnotetext{
${ }^{1}$ Grupo de Quadril, Departamento de Ortopedia e Traumatologia, Faculdade de Ciências Médicas da Santa Casa de São Paulo (FCMSCSP), Irmandade Santa Casa de Misericórdia, São Paulo, SP, Brasil

2 Laboratório de Análise do Movimento Humano, Departamento de Ciências da Reabilitação, Universidade Nove de Julho - UNINOVE, São Paulo, SP, Brasil
}

Rev Bras Ortop 2020;55(4):508.

\section{ERRATUM}

Rio de Janeiro, 01 de Agosto de2020

Caros leitores, No artigo É importante saber onde posicionar o marcador esférico para o planejamento digital de artroplastia de quadril? (Rev Bras Ortop 2020;55(03): 353-359. DOI: https:// doi.org/10.1055/s-0039-1693052.), publicado online na Revista Brasileira Ortopedia em Junho de 2020:

Onde se lê:

Giancarlo Cavalli Polesello ${ }^{1}$

Thiago Tronco Salerno ${ }^{1}$

João Hélio Zucarelli Rezende ${ }^{1}$

Marcelo Cavalheiro de Queiroz ${ }^{1}$

Nayra Deise Rabelo ${ }^{1,2}$

Walter Ricioli Junior $^{1}$

Leia-se:

Giancarlo Cavalli Polesello ${ }^{1}$

Thiago Tronco Salerno ${ }^{1}$

João Hélio Zucarelli Rezende ${ }^{1}$

Walter Ricioli Junior ${ }^{1}$

Nayra Deise Rabelo ${ }^{1,2}$

Marcelo Cavalheiro de Queiroz ${ }^{1}$ de Ortopedia e Traumatologia. Published by Thieme Revinter Publicações Ltda, Rio de Janeiro, Brazil 